\title{
Graft Versus Host Disease Prophylaxis/Therapy
}

National Cancer Institute

\section{Source}

National Cancer Institute. Graft Versus Host Disease Prophylaxis/Therapy. NCI

Thesaurus. Code C15448.

Therapy to prevent or treat graft vs host disease. 